\title{
Role of protein delta homolog 1 in the proliferation and differentiation of ameloblasts
}

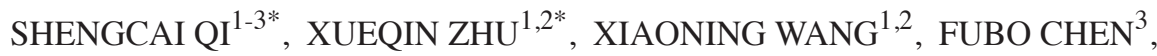 \\ YANHONG YAN ${ }^{4}$, GUANGWEI SHANG ${ }^{3}$ and WANTAO CHEN ${ }^{1,2}$
}

\begin{abstract}
${ }^{1}$ Department of Oral and Maxillofacial-Head and Neck Oncology, and Faculty of Oral and Maxillofacial Surgery, Ninth People's Hospital, Shanghai Jiao Tong University School of Medicine; ${ }^{2}$ Shanghai Research Institute of Stomatology and Shanghai Key Laboratory of Stomatology, Shanghai 200011; ${ }^{3}$ Department of Stomatology, Shanghai Tenth People's Hospital,

Tongji University, Shanghai 200072; ${ }^{4}$ Department of Pediatric Dentistry, School and Hospital of Stomatology, Tongji

University, Shanghai Engineering Research Center of Tooth Restoration and Regeneration, Shanghai 200072, P.R. China
\end{abstract}

Received May 30, 2017; Accepted October 12, 2017

DOI: $10.3892 / \mathrm{mmr} .2017 .8290$

\begin{abstract}
Protein delta homolog 1 (DLK1) regulates the odontoblastic differentiation of human dental pulp stem cells. It was hypothesized that DLK1 may exert regulatory effects on epithelial-mesenchymal interactions in tooth development. The present study investigated the expression of DLK1 during the development of mouse enamel and its role in the proliferation and differentiation of ameloblast-lineage cells (ALCs). DLK1 expression was upregulated in ameloblasts in the first mandibular molar during the entire process of enamel development. The mRNA and protein levels of DLK1 were significantly upregulated following ameloblastic induction in ALCs. In addition, overexpression of DLK1 promoted the proliferation of ALCs, inhibited ameloblastic differentiation, upregulated the expression of amelogenin and enamelin, and downregulated the expression of odontogenic ameloblast-associated protein and kallikrein 4 . The results of the present study suggested that DLK1 may be a potent regulator of ameloblast proliferation and differentiation, and may regulate enamel formation during tooth development.
\end{abstract}

Correspondence to: Dr Wantao Chen, Department of Oral and Maxillofacial-Head and Neck Oncology, and Faculty of Oral and Maxillofacial Surgery, Ninth People's Hospital, Shanghai Jiao Tong University School of Medicine, 639 Zhi Zao Ju Road, Shanghai 200011, P.R. China

E-mail: chenwantao196323@sjtu.edu.cn

Dr Guangwei Shang, Department of Stomatology, Shanghai Tenth People's Hospital, Tongji University, 299 Middle Yanchang Road, Shanghai 200072, P.R. China

E-mail: gwshang99@yahoo.com

*Contributed equally

Key words: protein delta homolog 1, enamel, ameloblast-lineage cell, proliferation, ameloblastic differentiation

\section{Introduction}

Tooth development is a highly conserved and coordinated process that controls tooth shape and size. Tooth morphogenesis and development is regulated by epithelial-mesenchymal interactions (1). The dentin layer is a type of mesenchymal-derived calcified tissue, which serves as the supporting tissue of the tooth. The enamel layer is a unique epithelium-derived calcified tissue, which covers the crown and forms the shape of the tooth in vertebrates. Dental enamel is composed of organized hydroxyapatite crystallites formed by the differentiation of ameloblasts (AMs), the differential activities of which may be divided into the initial secretory stage and maturation stage of amelogenesis (2). A number of factors regulate enamel-associated gene expression in AMs and dentin-associated gene expression in human dental pulp stem cells (hDPSCs). These factors include bone morphogenetic protein 2 (BMP2) (3), distal-less homeobox 3 (4), msh homeobox 2 (5), sonic hedgehog, Wnt (6) and neurogenic locus notch homolog protein (Notch) (7). It is meaningful to understand the molecular mechanisms that regulate the formation of enamel and dentin, which may be applicable to the prevention and treatment of heritable and acquired enamel diseases, including caries and enamel fluorosis (8), and to the replacement of enamel with biomaterials for therapeutic treatment.

The evolutionarily conserved Notch signaling pathway has a central role in numerous cellular processes during development and throughout adult life (9). In particular, the Notch pathway serves an essential role in enamel development (10), which is required for epithelial stem cell survival and enamel formation during the continuous growth of the mouse incisor (11). Protein delta homolog 1 (DLK1) is a transmembrane and secreted protein belonging to the Notch family of receptors and ligands (12). DLK1, as a Notch regulator, has been proven to be essential for normal development (13), which involves processes including neuroendocrine differentiation (14), hepatocyte and biliary epithelial cell differentiation (15), hematopoiesis (16), osteogenesis (17), skeletal muscle differentiation (18), and chondrogenic differentiation (19). DLK1 shares close structural similarities with 
periostin, which has additionally been proposed to serve a role in tooth development (20-22). A previous study demonstrated that DLK1 regulated the odontoblastic differentiation of hDPSCs (23). Therefore, it was hypothesized that DLK1 may exert regulatory effects on epithelial-mesenchymal interactions during tooth development. In particular, the present study investigated the potential roles of DLK1 in the proliferation and differentiation of AMs.

In the present study, DLK1 expression during enamel development in the molars of mice was detected by immunohistochemical analysis. In addition, DLK1 expression during ameloblast-lineage cell (ALC) differentiation was analyzed by reverse transcription-quantitative polymerase chain reaction (RT-qPCR) and western blot analyses, and DLK1 was stably overexpressed in ALCs to determine its effects on AM proliferation and differentiation.

\section{Materials and methods}

Ethics statement. The present study was performed according to an informed protocol approved by the Ethics Committee of the Ninth People's Hospital, Shanghai Jiao Tong University School of Medicine (Shanghai, China).

Immunohistochemical analysis. A total of nine adult C57BL/6J mice (3 male $22-24 \mathrm{~g}$ and 6 female approximately at 19-21 g; 8 -weeks-old) were mated overnight in a specific pathogen free animal center $\left(\sim 24^{\circ} \mathrm{C}, 60 \%\right.$ humidity, $10 \mathrm{~h}$ light $/ 14 \mathrm{~h}$ dark cycle, food and water was supplied ad libtum). The day on which a vaginal plug was observed was designated as embryonic day 0.5 (E0.5). A total of 3 embryos and 3 postnatal (PN) mice at each enamel developmental stage (E13.5, E16.5, E18.5, PN2 and PN6) were used in the present study. All the animals were deeply anesthetized by intraperitoneal injection of pentobarbital sodium $(60 \mathrm{mg} / \mathrm{kg})$ prior to sample collection, and all procedures and protocols used were in accordance with the Guidelines for Ethical Care of Experimental Animals (www.nap.edu/readingroom/books/labrats). Samples were prepared for immunohistochemistry by fixing the mandibles isolated at each stage in $4 \%$ paraformaldehyde overnight at $4^{\circ} \mathrm{C}$, followed by demineralization with $10 \%$ EDTA (pH 7.4) for 2-6 days for 2 weeks at $4^{\circ} \mathrm{C}$. Following dehydration and embedding in paraffin, the samples were sectioned to a thickness of $5 \mu \mathrm{m}$. The sections were dipped in xylene to remove the paraffin and rehydrated using a graded alcohol series $(100,95,85$ and $70 \%)$. The sections were incubated in $3 \%$ hydrogen peroxide for $10 \mathrm{~min}$ at room temperature to prevent endogenous peroxidase activity, incubated in $0.01 \mathrm{M}$ citrate for $10 \mathrm{~min}$ at $100^{\circ} \mathrm{C}$ and cooled at room temperature for $20 \mathrm{~min}$. The slides were subsequently blocked in $5 \%$ bovine serum albumin (Shanghai Weiao Biotechnology Co., Ltd. Shanghai, China) in PBS at $37^{\circ} \mathrm{C}$ for $30 \mathrm{~min}$. The slides were then incubated with a primary antibody against DLK1 (1:300; rabbit polyclonal antibody; cat. no. sc-8624; Santa Cruz Biotechnology, Inc., Dallas, TX, USA) overnight at $4^{\circ} \mathrm{C}$. A subset of slides was incubated with PBS as a negative control. The slides were washed with PBS and incubated with polymer helper and poly-horseradish peroxidase-anti-rabbit immunoglobulin G (OriGene Technologies, Inc., Beijing, China) for $1 \mathrm{~h}$ at $37^{\circ} \mathrm{C}$. Following counterstaining with hematoxylin at room temperature for $3 \mathrm{~min}$, the samples were visualized under a light microscope (magnification x200 and x400; Zeiss AG, Oberkochen, Germany).

Cell culture and differentiation assay. The ameloblast-like cell line ALC was provided by the Shanghai Research Institute of Stomatology and Shanghai Key Laboratory of Stomatology, Ninth People's Hospital, Shanghai Jiao Tong University (Shanghai, China). The cells were maintained in high-glucose Dulbecco's modified Eagle's medium (Gibco; Thermo Fisher Scientific, Inc., Waltham, MA, USA) containing $100 \mathrm{U} / \mathrm{ml}$ penicillin, $100 \mathrm{mg} / \mathrm{l}$ streptomycin and $10 \%$ fetal bovine serum (Gibco; Thermo Fisher Scientific, Inc.) at $37^{\circ} \mathrm{C}$ in a $5 \% \mathrm{CO}_{2}$ humidified atmosphere. Ameloblastic differentiation was induced in ALCs as described previously (2). ALCs were seeded at a density of $2 \times 10^{4}$ cells in six-well plates, and the medium was replaced with fresh medium supplemented with $20 \mathrm{mg} / \mathrm{l}$ retinoic acid (Sigma-Aldrich; Merck KGaA, Darmstadt, Germany) and $10^{-7} \mathrm{M}$ dexamethasone (Sigma-Aldrich; Merck $\mathrm{KGaA}$ ) when the cells reached $\sim 80 \%$ confluence. The medium was changed every 3 days. A subset of induced ALCs was used as a wild-type group in subsequent assays.

DLK1 lentiviral transfection. A lentivirus expressing green fluorescent protein (GFP) and harboring the DLK1 gene was constructed as the dlk1-overexpression (oe) group, while a lentivirus expressing GFP without the DLK1 gene was constructed as the control group (Shanghai GeneChem Co., Ltd., Shanghai, China). ALCs were seeded into six-well plates and grown to $\sim 50 \%$ confluence prior to transfection. The ALCs were infected with $10 \mu$ l lentiviruses $\left(1 \times 10^{8} \mathrm{U} / \mathrm{ml}\right)$ using polybrene reagent (Sigma-Aldrich; Merck KGaA) for $24 \mathrm{~h}$, according to the manufacturer's protocol. Following transfection, $10 \mu \mathrm{g} / \mathrm{ml}$ puromycin (Sigma-Aldrich; Merck KGaA) was added for 2 weeks to select the positively transfected cells. A monoclonal population of stably infected cells (termed dlk1-oe or control) were pooled and used for further experiments, respectively. The transfected cells were analyzed under a fluorescence microscope and confirmed by RT-qPCR and western blot analysis.

Cell proliferation assay. A Cell Counting Kit-8 (CCK-8; Dojindo Molecular Technologies, Inc., Kumamoto, Japan) assay was used to analyze the effect of DLK1 on ALC proliferation, according to the manufacturer's protocol. The control and dlk1-oe groups were seeded at a density of $2 \times 10^{3}$ cells/well into 96-well plates (5 wells/group; Corning Incorporated, Corning, NY, USA) and cultured for 1,3,5 and 7 days. The cell medium supplemented with $10 \%$ FBS was replenished daily. Following culturing, the number of cells was assessed using the CCK-8 assay. Absorbance was measured using a microplate reader at $450 \mathrm{~nm}$ to determine the number of viable cells in each well. A well containing medium and CCK-8 solution without cells was used as a blank control. Cell proliferation was represented as the mean \pm standard deviation of the absorbance of five wells for each group.

RNA isolation and RT-qPCR analysis. Total RNA was extracted from the wild-type, control and dlk1-oe groups cultured in ameloblastic induction medium for different 
durations (0, 3 and 7 days) using TRIzol reagent (Invitrogen; Thermo Fisher Scientific, Inc.), according to the manufacturer's protocol. cDNA was synthesized using a PrimeScript RT Reagent kit with gDNA Eraser (Takara Bio, Inc., Otsu, Japan) and used as a template for PCR. The ameloblastic differentiation of the cells was monitored by analyzing the expression of the following AM-related markers: Amelogenin (AMELX), enamelin, kallikrein 4 (KLK4), and matrix metallopeptidase 20 (MMP 20). GAPDH was used to normalize the expression of the target RNAs (2). The following primer sequences were used: AMELX forward, 5'-GATGGCTGC ACCACCAAATC-3' and reverse, 5'-CTGAAGGGTGTGACT CGGG-3'; enamelin forward, 5'-TGCAGAAATCCGACT TCTCCT-3' and reverse, 5'-CATCTGGAATGGCATGGC A-3'; KLK4 forward, 5'-CCGGATCATACAAGGCCAGG-3' and reverse, 5'-TGCGGATGCACCAAGACTC-3'; MMP 20 forward, 5'-CACCTCACAAGCCATCTATCC-3' and reverse, 5'-GAAGCTCCTTTCCCAACATTG-3'; DLK1 forward, 5'-CTCCCTGACTCTTGTTTG G-3' and reverse, 5'-AAC GGTGACAATGACTTGC-3'; and GAPDH forward, 5'-TGG GTGTGAACCATGAGAAGT-3' and reverse, 5'-TGAGTC CTTCCACGATACCAA-3'. The PCR reaction was performed with a Hieff ${ }^{\mathrm{TM}}$ qPCR SYBR ${ }^{\circledR}$ Green Master Mix (Shanghai Qcbio Science \& Technologies Co., Ltd., Shanghai, China) in an ABI 7500 RT-PCR System (Applied Biosystems; Thermo Fisher Scientific, Inc.). Amplification was performed under the following conditions: $95^{\circ} \mathrm{C}$ for $5 \mathrm{~min}$ in the holding stage; 40 cycles of $95^{\circ} \mathrm{C}$ for $10 \mathrm{sec}$ and $60^{\circ} \mathrm{C}$ for $30 \mathrm{sec}$ in the cycling stage; and $95^{\circ} \mathrm{C}$ for $15 \mathrm{sec}, 60^{\circ} \mathrm{C}$ for $1 \mathrm{~min}$ and $60^{\circ} \mathrm{C}$ for $15 \mathrm{sec}$ in the melt curve stage. Relative gene expression was calculated using the comparative $2^{-\Delta \Delta \mathrm{Cq}}$ method (24). The mean $\mathrm{Cq}$ value of the target gene was normalized to the averaged $\mathrm{Cq}$ values of GAPDH to obtain a $\Delta \mathrm{Cq}$ value, which was subsequently normalized to control samples to obtain a $\Delta \Delta \mathrm{Cq}$ value. Each measurement was assessed in triplicate. The gene expression ratio was presented as the mean \pm standard deviation of three independent experiments.

Western blot analysis. Cells in the control and dlk1-oe groups cultured in a 6-well plate under ameloblastic induction medium for 0,3 and 7 days were lysed with a protein extraction kit (Pierce; Thermo Fisher Scientific, Inc.), and cells were incubated for $15 \mathrm{~min}$ at a temperature of $4^{\circ} \mathrm{C}$ with gentle agitation every $5 \mathrm{~min}$. The lysate was collected and transferred to a microcentrifuge tube and centrifuged at $12,000 \mathrm{xg}$ for $10 \mathrm{~min}$ at $4^{\circ} \mathrm{C}$. The supernatant was collected and transferred into a new tube for analysis. Protein concentration was determined using a bicinchoninic acid protein assay kit (Pierce; Thermo Fisher Scientific, Inc.). An equal amount (30 $\mu \mathrm{g}$ per lane) of protein was separated via SDS-PAGE (6 and $10 \%$ gels, respectively) and transferred onto nitrocellulose membranes (EMD Millipore, Billerica, MA, USA). Following blocking with $5 \%$ milk at room temperature for $1 \mathrm{~h}$, the following primary antibodies were used overnight at $4^{\circ} \mathrm{C}$ : Rabbit anti-mouse DLK1 (1:1,000; cat. no. sc-8624; Santa Cruz Biotechnology, Inc.), rabbit anti-mouse AMELX (1:1,000; cat. no. sc-365284; Santa Cruz Biotechnology, Inc.), rabbit anti-mouse MMP 20 (1:1,000; cat. no. ab84737; Abcam, Cambridge, MA, USA), and rabbit anti-mouse GAPDH (1:5,000; cat. no. sc-47724; Santa Cruz Biotechnology, Inc.). Following three washes with TBS containing Tween 20, membranes were incubated with goat anti-mouse immunoglobulin G (1:10,000; cat. no. 926-32210, LI-COR Biosciences, Lincoln, NE, USA) and goat anti-rabbit immunoglobulin G (1:10,000; cat. no. 926-32211, LI-COR Biosciences) secondary antibodies for $1 \mathrm{~h}$ at room temperature with agitation. Subsequent to the final wash, the membranes were visualized using an Odyssey LI-COR system (LI-COR Biosciences) and analyzed using an Odyssey infrared imaging system (LI-COR Biosciences, application software version 3.0).

Alkaline phosphatase (ALP) staining. The wild-type, control and dlk1-oe groups were seeded $5 \times 10^{5}$ cells/per well in 6-well plates in triplicate and cultured in ameloblastic induction medium for 7 days. The induction medium was replaced every 3 days. An ALP color development kit (Beyotime Institute of Biotechnology, Haimen, China) was used, according to the manufacturer's protocol. The plates were treated with an ALP staining kit for $30 \mathrm{~min}$ at $37^{\circ} \mathrm{C}$ following fixation in $4 \%$ paraformaldehyde for $15 \mathrm{~min}$ at $37^{\circ} \mathrm{C}$. The cells were washed three times with distilled water and observed by phase-contrast microscopy (magnification x200).

Statistical analysis. Experiments were performed in triplicate, and data are presented as the mean \pm standard deviation. Data were evaluated by one-way analysis of variance followed by a Tukey's post hoc test using SPSS software (version 10.0; SPSS, Inc., Chicago, IL, USA). $\mathrm{P}<0.05$ was considered to indicate a statistically significant difference.

\section{Results}

Location of DLK1 protein during mouse molar development. DLK1 protein expression in the front sections of the first mandibular molar tooth germs in mice was assessed by immunohistochemical analysis. At the early bud stage (E13.5), DLK1 was strongly expressed in the dental epithelium. Positive immunolabelling was observed in the basal epithelial cells and surrounding mesenchymal cells (Fig. 1A). At the early bell stage (E16.5), positive immunolabelling was observed in the inner enamel epithelium (IEE), particularly in the cervical loop (CL) and enamel knot (EK) (Fig. 1B). At the late bell stage (E18.5), DLK1 was expressed at a low level in the dental pulp (DP) cells located near the odontoblasts (ODs), IEE and outer enamel epithelium (OEE). However, DLK1 was strongly expressed in the ODs, stellate intermediate (SI) layer and stellate reticulum (SR) adjacent to the SI layer (Fig. 1C) during the late bell stage. At the PN2 stage, DLK1 was located in the AMs and strongly expressed in DP cells located near the ODs, SI layer and SR adjacent to the SI layer (Fig. 1D). At the PN6 stage, DLK1 was strongly expressed in the ODs, AMs and SR adjacent to the AMs (Fig. 1E). Therefore, positive expression of DLK1 was observed in the AMs and ODs during development, particularly in the AMs located near the enamel and ODs located near the dentin.

DLK1 and AM-associated gene expression during ameloblastic differentiation of ALCs. In the present study, DLK1 expression was detected to evaluate its physiological significance during ameloblastic differentiation. Mineralization-associated genes were measured to directly 

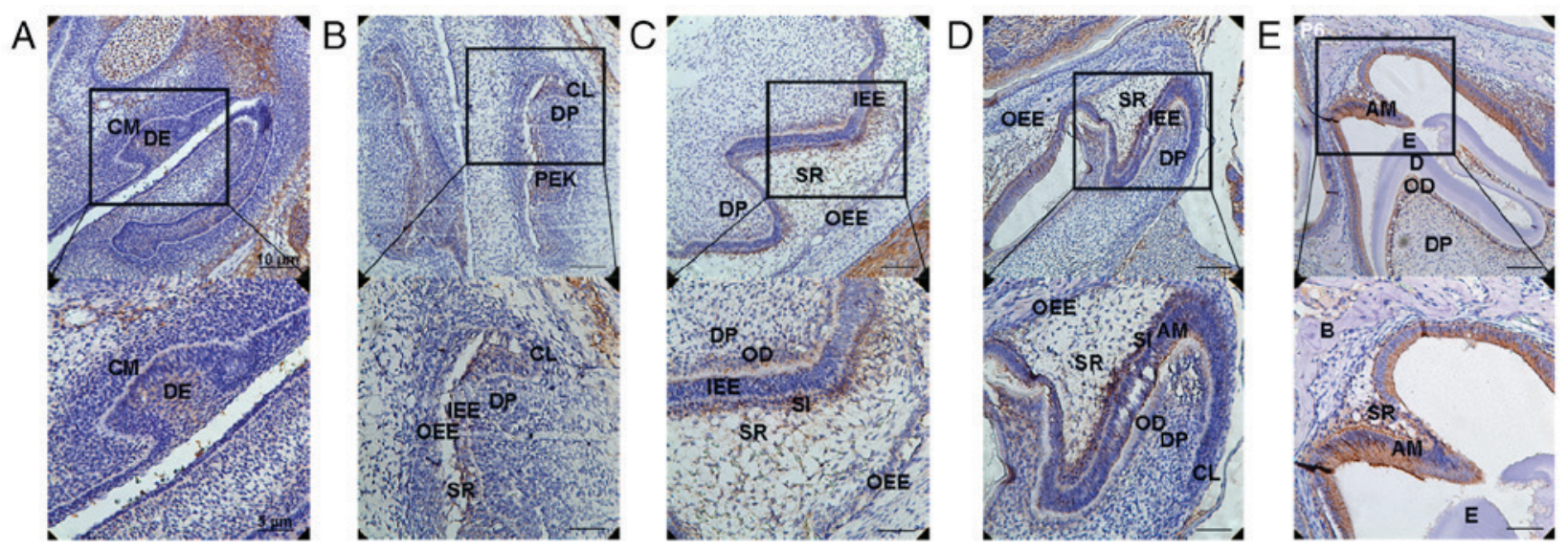

Figure 1. Expression of DLK1 during enamel development. (A) DLK1 was strongly expressed in the DE during the early bud stage (E13.5). Positive immunolabelling was observed in the basal epithelial cells and surrounding mesenchymal cells. (B) Positive immunolabelling was also observed in the IEE, particularly in the CL and EK during the early bell stage (E16.5). (C) At the late bell stage (E18.5), DLK1 was expressed at a low level in the DP cells located near the ODs, IEE and OEE. Primarily, DLK1 was strongly expressed in the ODs, the SI layer and SR adjacent to the SI layer. (D) During PN2, DLK1 was located in the AMs and strongly expressed in the DP cells located near the ODs, SI layer and SR adjacent to the SI layer. (E) During PN6, DLK1 was strongly expressed in the ODs, AMs and SR adjacent to the AMs. Upper panels, magnification x200; lower panels, magnification, x400. DE, dental epithelium; IEE, inner enamel epithelium; CL, cervical loop; EK, enamel knot; OD, odontoblast; OEE, outer enamel epithelium; SI, stellate intermediate; ST, stellate reticulum; E, embryonic development stage; DP, dental pulp; DLK1, protein delta homolog 1; AM, ameloblast; PN, postnatal day.
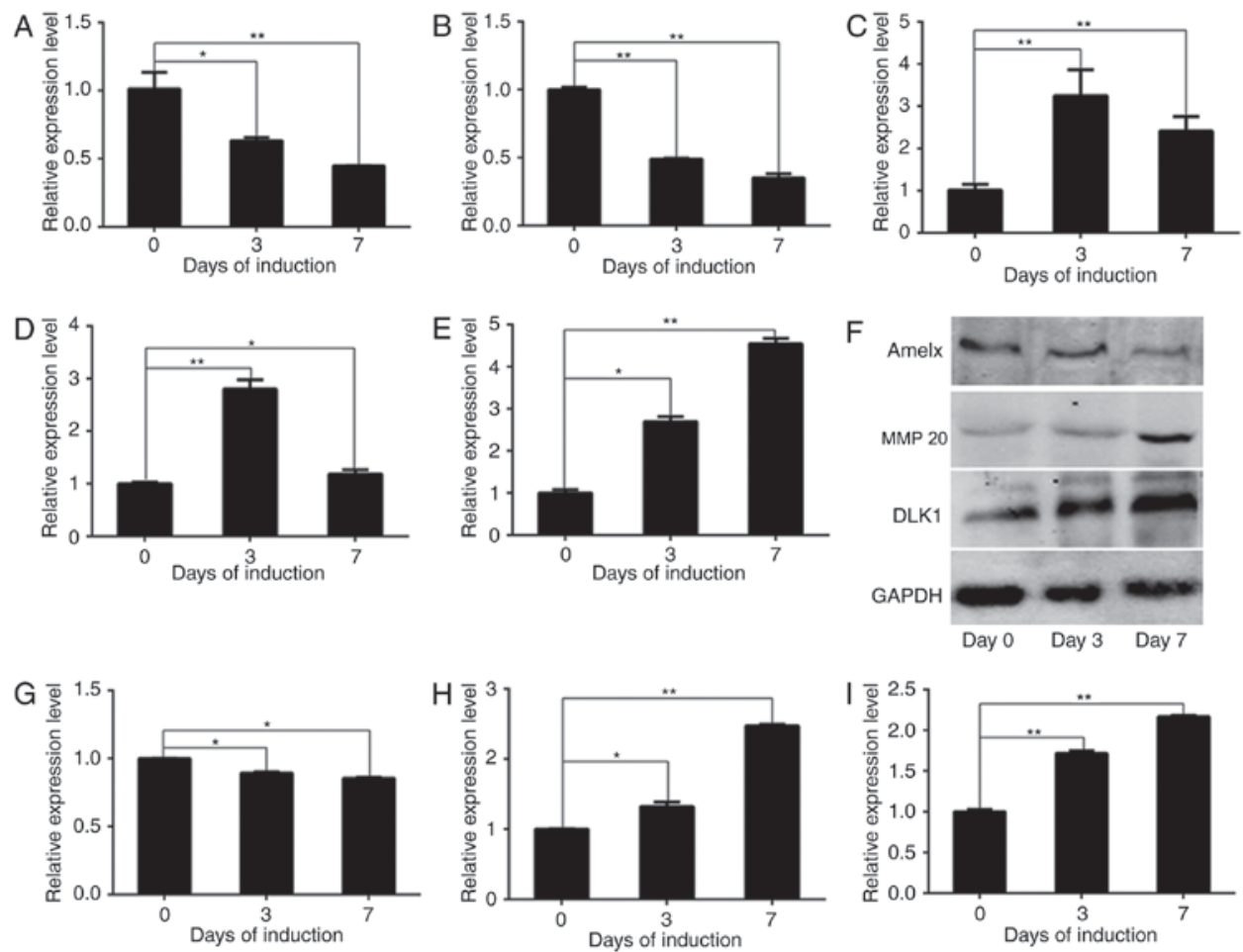

Figure 2. DLK1 expression during the ameloblastic differentiation of ameloblast-lineage cells. The mRNA levels of AMELX, enamelin, MMP 20, KLK4 and DLK1 are presented, in addition to the protein levels of AMELX, MMP 20 and DLK1 during ameloblastic induction. Quantified results of the western blot analysis based on measurements of optical density are presented, and the data represent the mean \pm standard deviation of three independent experiments. The mRNA levels of (A) AMELX and (B) enamelin decreased significantly, whereas those of (C) MMP 20 (D) KLK4 increased significantly. (E) The mRNA levels of DLK1 increased gradually. The protein levels of AMELX and MMP 20 exhibited the same trends as their mRNA levels, as demonstrated by (F) western blot analysis. The results of the western blot analysis of (G) AMELX, (H) MMP 20 and (I) DLK1 are presented. These results demonstrated that ameloblastic differentiation was successfully induced in vitro. ${ }^{*} \mathrm{P}<0.05 ;{ }^{* * *} \mathrm{P}<0.01$. DLK1, protein delta homolog 1; AMELX, amelogenin; MMP 20, matrix metallopeptidase 20; KLK4, kallikrein 4.

verify the functional role of DLK1 in ameloblastic differentiation. In order to analyze the expression of AM-associated genes and DLK1 during ameloblastic induction, the mRNA levels of AMELX, enamelin, MMP 20, KLK4 and DLK1 were detected in wild-type ALCs by RT-qPCR analysis, and the protein levels of AMELX, MMP 20 and DLK1 were detected by western blot analysis (Fig. 2). The mRNA levels of AMELX (Fig. 2A) [day (D)0 vs. D3, P=0.029; D0 vs. D7, P=0.0072] and 
A

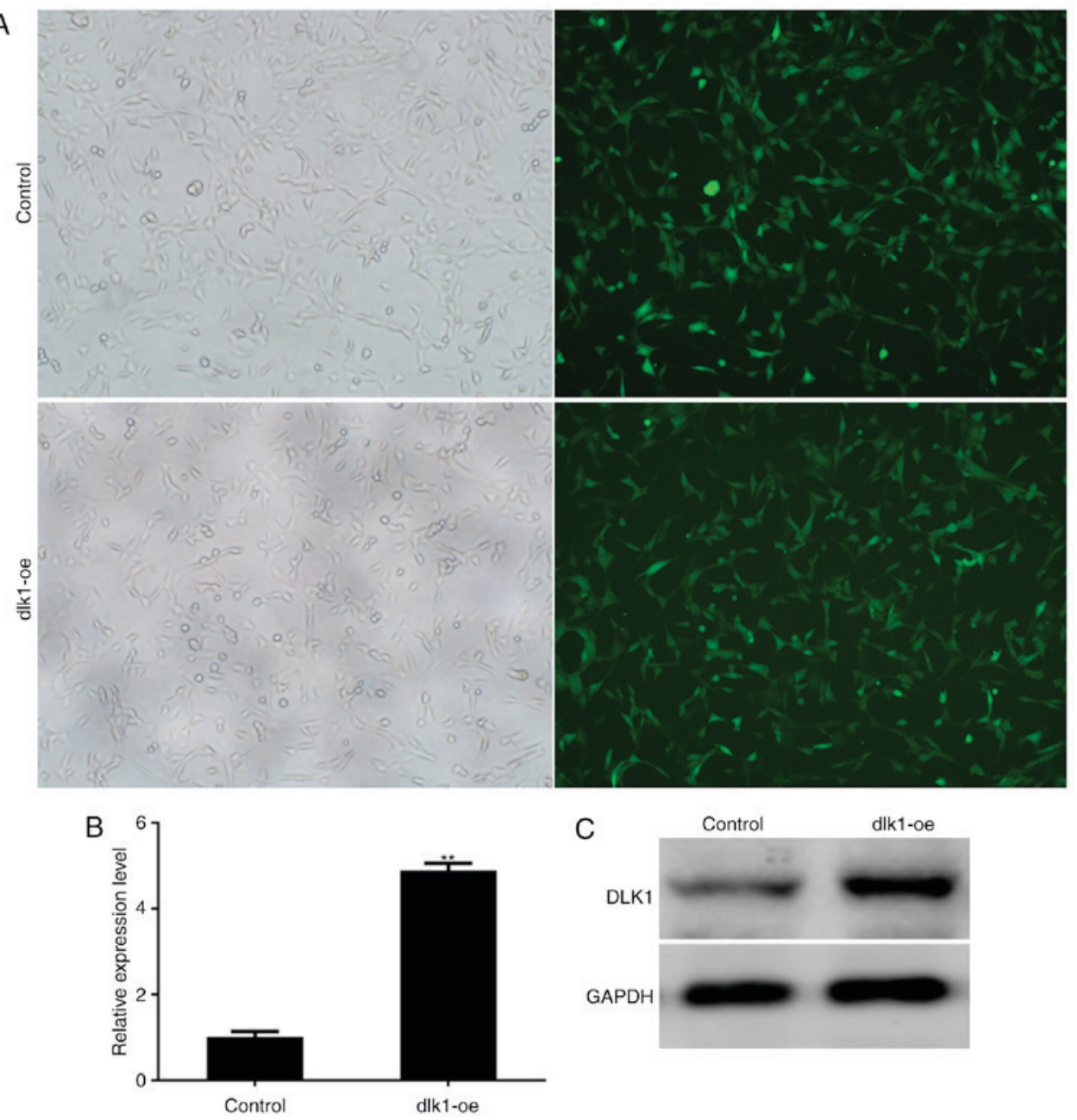

Figure 3. Stable overexpression of DLK1 in ALCs. (A) ALCs transfected with lentiviral vector in vitro exhibited green fluorescent protein expression (magnification, $\mathrm{x} 200$ ). The (B) mRNA and (C) protein levels of DLK1 in the control and dlk1-oe groups, indicating that DLK1 was overexpressed in the dlk1-oe group ${ }^{* *} \mathrm{P}<0.01$ vs. control. DLK1, protein delta homolog 1; oe, overexpression; ALC, ameloblast-lineage cell.

enamelin (Fig. 2B) (D0 vs. D3, P=0.0089; D0 vs. D7, P=0.0012) were downregulated, whereas those of MMP 20 (Fig. 2C) (D0 vs. D3, P=0.0049; D0 vs. D7, P=0.0072) and KLK4 (Fig. 2D) (D0 vs. D3, $\mathrm{P}=0.0031$; D0 vs. $\mathrm{D} 7, \mathrm{P}=0.0324$ ) were upregulated gradually. The protein levels of AMELX (Fig. 2G) (D0 vs. D3, $\mathrm{P}=0.0369$; $\mathrm{D} 0$ vs. $\mathrm{D} 7, \mathrm{P}=0.0352$ ) and MMP 20 (Fig. $2 \mathrm{H}$ ) (D0 vs. D3, $\mathrm{P}=0.0273$; $\mathrm{D} 0$ vs. $\mathrm{D} 7, \mathrm{P}=0.0002$ ) followed the same trends as their mRNA expression during ameloblastic induction (Fig. 2F-H). Additionally, the mRNA and protein levels of DLK1 were upregulated during ameloblastic differentiation, as presented in Fig. 2E (D0 vs. D3, $\mathrm{P}=0.0119$; D0 vs. D7, $\mathrm{P}=0.0022$ ), and Fig. $2 \mathrm{~F}$ and I (D0 vs. D3, $\mathrm{P}=0.0072 ; \mathrm{D} 0$ vs. $\mathrm{D} 7, \mathrm{P}=0.0033$ ).

Stable overexpression of DLK1 in ALCs. ALCs were transfected with a lentiviral vector expressing GFP alone (control group) or a lentiviral vector expressing DLK1 and GFP (dlk1-oe group) in vitro, and exhibited GFP expression within 3 days of transduction (Fig. 3A). The expression of DLK1 in stably transfected cells was subsequently assessed. The mRNA and protein levels of DLK1 were markedly increased in the dlk1-oe group compared with the control group following infection (Fig. 3B and C; $\mathrm{P}=0.0019)$. These results demonstrated that stable overexpression of DLK1 was successfully established in ALCs following lentiviral transfection.

DLK 1 promotes the proliferation of ALCs. Cell proliferation was measured using a CCK-8 assay, according to the manufacturer's protocol. The results indicated that the rate of cell proliferation in the dlk1-oe group was markedly increased compared with that of the control group on days $1(\mathrm{P}=0.031)$, $3(\mathrm{P}=0.042), 5(\mathrm{P}=0.0053)$ and $7(\mathrm{P}=0.018)$ (Fig. 4A). These results demonstrated that cell proliferation was promoted in the dlk1-oe group.

Inhibition of ameloblastic differentiation in ALCs following DLK1 overexpression. The mRNA levels of AMELX (Fig. 4B; $\mathrm{P}=0.0413, \mathrm{P}=0.0028$ and $\mathrm{P}=0.0091$ ) and enamelin (Fig. 4C; $\mathrm{P}=0.0013, \mathrm{P}=0.048$ and $\mathrm{P}=0.0001)$ in the dlk1-oe group were significantly increased compared with those in the control group following 7 days of culture in induction medium. In addition, the mRNA levels of MMP 20 (Fig. 4D; $\mathrm{P}=0.0029, \mathrm{P}=0.0068$ and $\mathrm{P}=0.0054$ ) and KLK4 (Fig. 4E; $\mathrm{P}=0.0093, \mathrm{P}=0.0056$ and $\mathrm{P}=0.0027$ ) in the dlk1-oe group were significantly decreased compared with those in the control group. Similar trends in the protein expression levels of MMP 20 (Fig. 4F; $\mathrm{P}=0.0073$, 

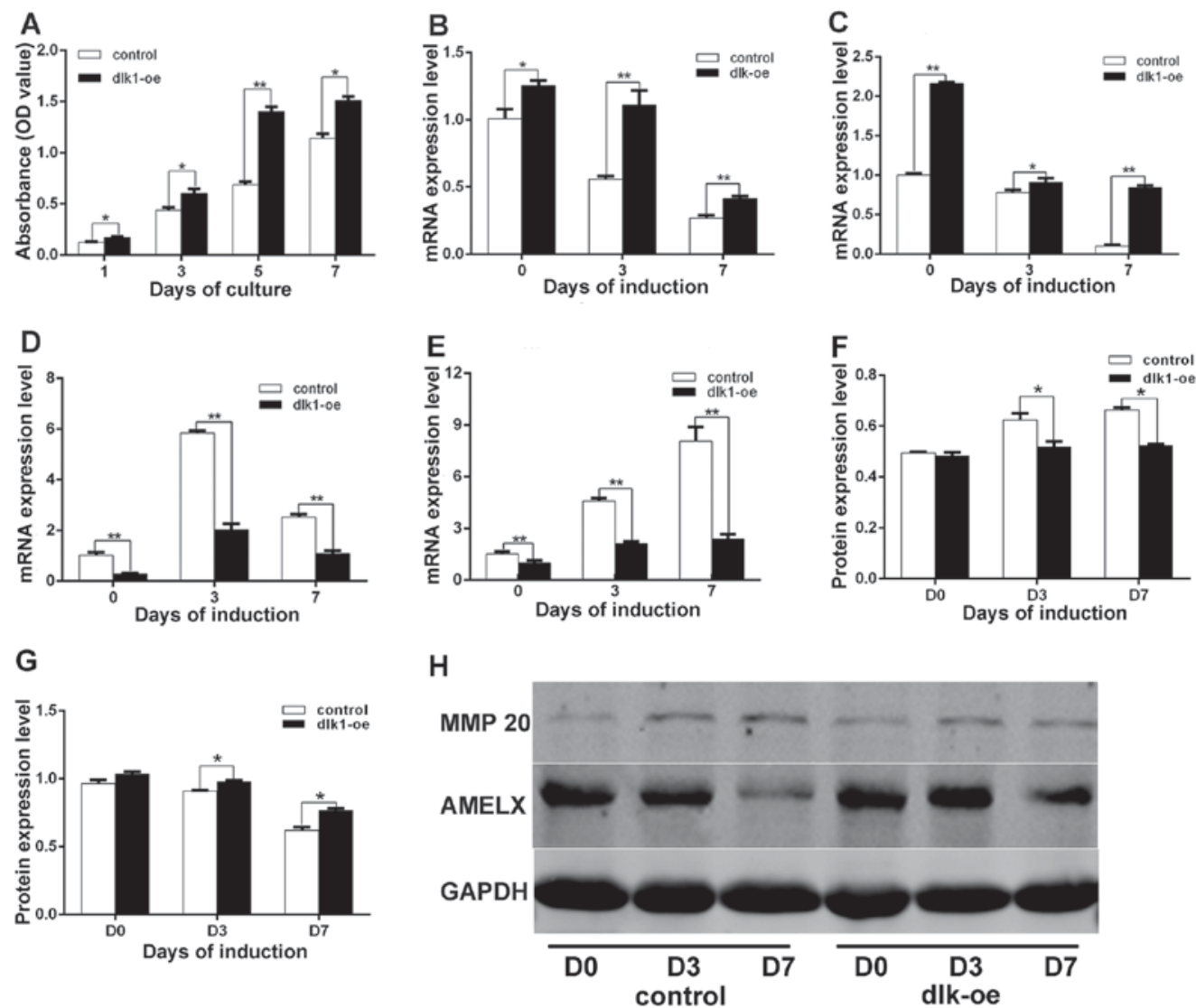

Figure 4. Effects of DLK1 on ALC proliferation and differentiation. (A) The stimulatory effect of DLK1 on ALC proliferation. A CCK-8 assay indicated that the rate of cell proliferation in the dlk1-oe group was markedly increased compared with the control group on days 1, 3, 5 and 7 . The mRNA levels of (B) AMELX, (C) enamelin, (D) MMP 20 and (E) KLK4 were measured following DLK1 overexpression in ALCs during ameloblastic differentiation, which indicated that DLK1 overexpression upregulated AMELX and enamelin mRNA, while downregulating the mRNA levels of MMP 20 and KLK4. The quantified protein expression data for (F) MMP 20 and (G) AMELX were calculated based on measurements of the optical density value. The protein levels of AMELX and MMP 20 followed the same trend as their mRNA levels. (H) The protein levels of MMP 20 and AMELX were measured by western blot analysis. All data are presented as the mean \pm standard deviation of at least three independent experiments. ${ }^{*} \mathrm{P}<0.05 ;{ }^{* * *} \mathrm{P}<0.01$. DLK1, protein delta homolog 1; AMELX, amelogenin; MMP 20, matrix metallopeptidase 20; KLK4, kallikrein 4; ALC, ameloblast-lineage cell; D, day; oe, overexpression.

$\mathrm{P}=0.025$ and $\mathrm{P}=0.039$ ) and AMELX (Fig. 4G; $\mathrm{P}=0.0063$, $\mathrm{P}=0.0045$ and $\mathrm{P}=0.0024)$ were observed in the dlk1-oe and control groups via western blot analysis, and the western blot results are illustrated in Fig. 4H. Additionally, ALP staining was markedly reduced in the wild-type and control groups on day 7 when compared with the dlk1-oe group (Fig. 5). These results indicated that DLK1 overexpression inhibited the ameloblastic differentiation of ALCs.

\section{Discussion}

Tooth development requires continuous interaction and cross-talk between various epithelial and mesenchymal cells, which involves different growth factors and signaling molecules. DLK1 exerts regulatory effects on cell differentiation and controls various signaling pathways during normal development. Numerous heritable defects, including growth retardation, obesity and skeletal malformations, have been observed in DLK1-null mice, and symptoms of maternal uniparental disomy syndrome have been associated with DLK1 deficiency in humans $(25,26)$. DLK1 is a negative regulator of a number of differentiation processes, including neuroendocrine differentiation (14), osteogenesis, chondrogenesis (19)

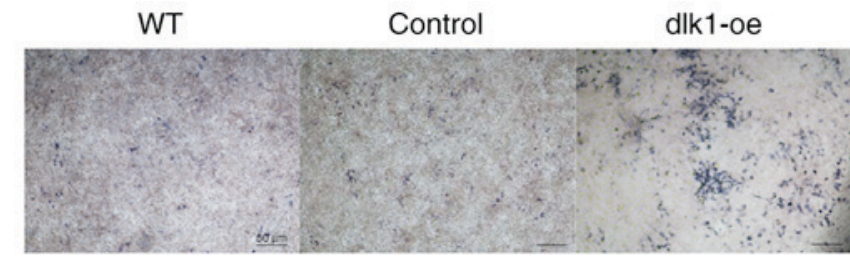

Figure 5. ALP staining results following DLK1 overexpression in ALCs. The results demonstrated that ALP staining in the WT and control groups was markedly stronger compared with the dlk1-oe group (magnification, $\mathrm{x} 100$ ), which suggested that DLK1 overexpression inhibited ALC ameloblastic differentiation. ALP, alkaline phosphatase; DLK1, protein delta homolog 1; ALC, ameloblast-lineage cell; oe, overexpression; WT, wild-type.

and muscle regeneration (17). However, the in vivo and in vitro effects of DLK1 on ameloblastic proliferation and differentiation remain unclear.

During embryonic development, DLK1 is expressed at a high level in a number of tissues, including the lungs, adrenal cortex, proximal tubules of the kidneys and mesoderm-derived tissues, including chondroblast tissue (19) and skeletal myotubes (14). A previous study demonstrated that DLK1 may 
regulate the development of mesoderm-derived dentin tissue in mice (23). In the present study, DLK1 expression was upregulated between E13.5 and E18.5 in the inner and outer enamel epithelium, and was markedly expressed in the AMs at stage PN6 following enamel formation. These results demonstrated that DLK1 may serve a regulatory role in the development of epithelium-derived enamel tissue, ultimately suggesting that DLK1 serves important roles in tooth development through epithelial-mesenchymal interactions. Therefore, it is useful to analyze the function of DLK1 during AM proliferation and differentiation.

Mouse ALCs, based on a previous study (2), which express secretory-stage and maturation-stage-associated genes, were selected to verify the effects of DLK1 on ameloblastic differentiation and proliferation in vitro. Proteins of the enamel matrix (enamelin, AMELX, MMP 20 and KLK4) are essential for enamel formation and may be used as mineralization markers to assess the ameloblastic differentiation of ALCs (2). In the present study, enamelin and AMELX were downregulated during the ameloblastic differentiation of ALCs, while MMP 20 and KLK4 were upregulated. The variations in these markers during the present experiments exhibited the same trends as in previous reports (2), and demonstrated that the ALCs underwent differentiation into a more mature stage during the course of the study; additionally, the mRNA and protein levels of DLK1 were significantly upregulated during this process.

Lentiviral vectors were used to achieve efficient gene overexpression in vitro. Results of the CCK-8 assay demonstrated that cell proliferation in the Dlk1-oe group was significantly upregulated. No significant differences were observed between the wild-type and control groups in terms of proliferation and differentiation. These findings indicated that recombinant lentiviral infection did not disturb cellular properties. Notably, DLK1 overexpression promoted the proliferation of ALCs, an effect that has previously been documented in lung epithelial cell- and pluripotent stem cell-derived neural progenitors $(27,28)$.

In addition, the ameloblastic differentiation markers enamelin, AMELX, MMP 20 and KLK4 were observed to be regulated in the dlk1-oe group, as demonstrated by RT-qPCR and western blot analyses. The mRNA levels of enamelin and AMELX, and the protein levels of AMELX, were upregulated in the dlk1-oe group during ameloblastic differentiation compared with the control group. By contrast, the protein and mRNA levels of MMP 20, and the mRNA level of KLK4, were downregulated in the dlk1-oe group. These results confirmed that DLK1 overexpression inhibited the ameloblastic differentiation of ALCs. Immunohistochemistry of DLK1 expression during tooth germ development demonstrated that DLK1 was highly expressed in the EK, which serves as a signaling center during the formation and regulation of tooth shape and size (29), at stage E16.5. DLK1 was additionally highly expressed in the AMs following enamel formation at PN6. These results suggested that DLK1 may inhibit AM differentiation to prevent excessive enamel formation.

DLK1, a Notch regulator, is able to activate Notch signaling, which serves important roles in the differentiation of odontoblasts and osteoblasts, calcification of hard tooth tissues, formation of cusp patterns and generation of tooth roots (30). Additionally, DLK1 activates human stem cells by interrupting the sonic hedgehog signaling pathway (31). DLK1 additionally activates the mitogen-activated protein kinase (MAPK) and MAPK kinase (MEK)/extracellular signal-regulated kinase (ERK) pathways to inhibit adipogenesis and chondrogenesis $(19,32,33)$. In addition, DLK1 regulates osteogenesis and chondrogenesis through phosphatidylinositol 3-kinase $(\mathrm{PI} 3 \mathrm{~K}) / \mathrm{RAC}-\alpha$ serine/threonine-protein kinase (AKT) signaling (19). Notably, tooth development involves a number of overlapping pathways, including the MAPK (34), MEK/ERK (34) and PI3K/AKT (35) pathways, which are associated with epithelial-mesenchymal interactions. The development of enamel and dentin involves processes similar to those in osteogenesis and chondrogenesis. Therefore, it was hypothesized that DLK1 may be involved in enamel development. Further intensive studies are required to elucidate the molecular cross-talk and signaling pathways associated with DLK1 in dentin and enamel development.

In conclusion, the present study detected the expression of DLK1 during the entire process of tooth development. DLK1 overexpression resulted in the increased proliferation capacity of AMs and regulated the expression of enamel mineralization-associated genes and proteins. Thus, DLK1 may serve a role in enamel development.

\section{Acknowledgements}

The present study was supported by grants from the National Natural Science Foundation of China (grant nos. 81500806 and 31271341), the China Postdoctoral Science Foundation (grant no. 2015M581633), and the Science Foundation of Shanghai Health and Family Planning Commission (grant no. 20144Y0257).

\section{References}

1. Mina M and Kollar EJ: The induction of odontogenesis in non-dental mesenchyme combined with early murine mandibular arch epithelium. Arch Oral Biol 32: 123-127, 1987.

2. Sarkar J, Simanian EJ, Tuggy SY, Bartlett JD, Snead ML, Sugiyama T and Paine ML: Comparison of two mouse ameloblast-like cell lines for enamel-specific gene expression. Front Physiol 5: 277, 2014.

3. Guo F, Feng J, Wang F, Li W, Gao Q, Chen Z, Shoff L, Donly KJ, Gluhak-Heinrich J, Chun YH, et al: Bmp2 deletion causes an amelogenesis imperfecta phenotype via regulating enamel gene expression. J Cell Physiol 230: 1871-1882, 2015.

4. Price JA, Wright JT, Walker SJ, Crawford PJ, Aldred MJ and Hart TC: Tricho-dento-osseous syndrome and amelogenesis imperfecta with taurodontism are genetically distinct conditions. Clin Genet 56: 35-40, 1999.

5. Zhou YL, Lei Y and Snead ML: Functional antagonism between Msx2 and CCAAT/enhancer-binding protein alpha in regulating the mouse amelogenin gene expression is mediated by protein-protein interaction. J Biol Chem 275: 29066-29075, 2000.

6. Liu F, Chu EY, Watt B, Zhang Y, Gallant NM, Andl T, Yang SH, Lu MM, Piccolo S, Schmidt-Ullrich R, et al: Wnt/beta-catenin signaling directs multiple stages of tooth morphogenesis. Dev Biol 313: 210-224, 2008.

7. Mitsiadis TA, Graf D, Luder H, Gridley T and Bluteau G: BMPs and FGFs target Notch signalling via jagged 2 to regulate tooth morphogenesis and cytodifferentiation. Development 137: 3025-3035, 2010.

8. Riksen EA, Kalvik A, Brookes S, Hynne A, Snead ML, Lyngstadaas SP and Reseland JE: Fluoride reduces the expression of enamel proteins and cytokines in an ameloblast-derived cell line. Arch Oral Biol 56: 324-330, 2011. 
9. Artavanis-Tsakonas S, Rand MD and Lake RJ: Notch signaling Cell fate control and signal integration in development. Science 284: 770-776, 1999.

10. Mitsiadis TA, Regaudiat L and Gridley T: Role of the Notch signalling pathway in tooth morphogenesis. Arch Oral Biol 50: $137-140,2005$

11. Felszeghy S, Suomalainen M and Thesleff I: Notch signalling is required for the survival of epithelial stem cells in the continuously growing mouse incisor. Differentiation 80: 241-248, 2010.

12. Traustadóttir GÁ, Jensen $\mathrm{CH}$, Thomassen M, Beck HC Mortensen SB, Laborda J, Baladrón V, Sheikh SP and Andersen DC: Evidence of non-canonical NOTCH signaling: Delta-like 1 homolog (DLK1) directly interacts with the NOTCH1 receptor in mammals. Cell Signal 28: 246-254, 2016.

13. Falix FA, Aronson DC, Lamers WH and Gaemers IC: Possible roles of DLK1 in the Notch pathway during development and disease. Biochim Biophys Acta 1822: 988-995, 2012.

14. Floridon C, Jensen CH, Thorsen P, Nielsen O, Sunde L, Westergaard JG, Thomsen SG and Teisner B: Does fetal antigen 1 (FA1) identify cells with regenerative, endocrine and neuroendocrine potentials? A study of FA1 in embryonic, fetal, and placental tissue and in maternal circulation. Differentiation 66: 49-59, 2000.

15. Tanimizu N, Nishikawa M, Saito H, Tsujimura T and Miyajima A Isolation of hepatoblasts based on the expression of Dlk/Pref-1. J Cell Sci 116: 1775-1786, 2003.

16. Moore KA, Pytowski B, Witte L, Hicklin D and Lemischka IR Hematopoietic activity of a stromal cell transmembrane protein containing epidermal growth factor-like repeat motifs. Proc Nat Acad Sci USA 94: 4011-4016, 1997.

17. Abdallah BM, Jensen CH, Gutierrez G, Leslie RG, Jensen TG and Kassem M: Regulation of human skeletal stem cells differentiation by Dlk1/Pref-1. J Bone Miner Res 19: 841-852, 2004.

18. Andersen DC, Petersson SJ, Jørgensen LH, Bollen P, Jensen PB, Teisner B, Schroeder HD and Jensen CH: Characterization of DLK1+ cells emerging during skeletal muscle remodeling in response to myositis, myopathies, and acute injury. Stem Cells 27: 898-908, 2009.

19. Chen L, Qanie D, Jafari A, Taipaleenmaki H, Jensen $\mathrm{CH}$, Säämänen AM, Sanz ML, Laborda J, Abdallah BM and Kassem M: Delta-like 1/fetal antigen-1 (Dlk1/FA1) is a novel regulator of chondrogenic cell differentiation via inhibition of the Akt kinase-dependent pathway. J Biol Chem 286: 32140-32149, 2011.

20. Li H, Marijanovic I, Kronenberg MS, Erceg I, Stover ML, Velonis D, Mina M, Heinrich JG, Harris SE, Upholt WB, et al: Expression and function of Dlx genes in the osteoblast lineage. Dev Biol 316: 458-470, 2008.

21. Hassan MQ, Saini S, Gordon JA, van Wijnen AJ, Montecino M, Stein JL, Stein GS and Lian JB: Molecular switches involving homeodomain proteins, HOXA10 and RUNX2 regulate osteoblastogenesis. Cells Tissues Organs 189: 122-125, 2009.

22. Ma D, Zhang R, Sun Y, Rios HF, Haruyama N, Han X, Kulkarni AB, Qin C and Feng JQ: A novel role of periostin in postnatal tooth formation and mineralization. J Biol Chem 286: 4302-4309, 2011.
23. Qi S, Yan Y, Wen Y, Li J, Wang J, Chen F, Tang X, Shang G, $\mathrm{Xu}$ Y and Wang R: The effect of delta-like 1 homolog on the proliferation and odontoblastic differentiation in human dental pulp stem cells. Cell Prolif 50, 2017.

24. Livak KJ and Schmittgen TD: Analysis of relative gene expression data using real-time quantitative PCR and the 2(-Delta Delta $\mathrm{C}(\mathrm{T})$ ) method. Methods 25: 402-408, 2001

25. Cheung LY, Rizzoti K, Lovell-Badge R and Le Tissier PR: Pituitary phenotypes of mice lacking the notch signalling ligand delta-like 1 homolog. J Neuroendocrinol 25: 391-401, 2013.

26. Mortensen SB, Jensen CH, Schneider M, Thomassen M, Kruse TA, Laborda J, Sheikh SP and Andersen DC: Membrane-tethered delta-like 1 homolog (DLK1) restricts adipose tissue size by inhibiting preadipocyte proliferation. Diabetes 61: 2814-2822, 2012.

27. Weng T, Gao L, Bhaskaran M, Guo Y, Gou D, Narayanaperumal J, Chintagari NR, Zhang K and Liu L: Pleiotrophin regulates lung epithelial cell proliferation and differentiation during fetal lung development via beta-catenin and Dlk1. J Biol Chem 284: 28021-28032, 2009.

28. Surmacz B, Noisa P, Risner-Janiczek JR, Hui K, Ungless M, Cui $\mathrm{W}$ and Li M: DLK1 promotes neurogenesis of human and mouse pluripotent stem cell-derived neural progenitors via modulating Notch and BMP signalling. Stem Cell Rev 8: 459-471, 2012

29. Mustonen T, Tümmers M, Mikami T, Itoh N, Zhang N, Gridley T and Thesleff I: Lunatic fringe, FGF, and BMP regulate the Notch pathway during epithelial morphogenesis of teeth. Dev Biol 248: 281-293, 2002

30. Cai X, Gong P, Huang Y and Lin Y: Notch signalling pathway in tooth development and adult dental cells. Cell Prolif 44: 495-507, 2011.

31. Han Z, Yu C, Tian Y, Zeng T, Cui W, Mager J and Wu Q: Expression patterns of long noncoding RNAs from Dlk1-Dio3 imprinted region and the potential mechanisms of Gt12 activation during blastocyst development. Biochem Biophys Res Commun 463: 167-173, 2015 .

32. Kim KA, Kim JH, Wang Y and Sul HS: Pref-1 (preadipocyte factor 1) activates the MEK/extracellular signal-regulated kinase pathway to inhibit adipocyte differentiation. Mol Cell Biol 27: 2294-2308, 2007.

33. Kim SW, Muise AM, Lyons PJ and Ro HS: Regulation of adipogenesis by a transcriptional repressor that modulates MAPK activation. J Biol Chem 276: 10199-10206, 2001.

34. Greenblatt MB, Kim JM, Oh H, Park KH, Choo MK, Sano Y, Tye CE, Skobe Z, Davis RJ, Park JM, et al: p38 $\alpha$ MAPK is required for tooth morphogenesis and enamel secretion. J Biol Chem 290: 284-295, 2015.

35. Sakoda K, Nakajima Y and Noguchi K: Enamel matrix derivative induces production of vascular endothelial cell growth factor in human gingival fibroblasts. Eur J Oral Sci 120: 513-519, 2012.

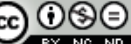

This work is licensed under a Creative Commons Attribution-NonCommercial-NoDerivatives 4.0 International (CC BY-NC-ND 4.0) License. 